\section{Revista Brasileira de Administração Científica}

Brazilian Journal of Scientific Administration

Jan a Mar 2021 - v.12 - n.1

\title{
Uma análise dos custos e internações por acidente vascular cerebral no Nordeste, 2008-2019
}

Existe um importante subfinanciamento do Sistema Único de Saúde e uma falta de informações acerca dos custos e demandas em saúde, dados fundamentais para gestores em saúde possam realizar as tomadas de decisão. Analisar as internações por AVC no SUS na região Nordeste, durante o período de 2008 a 2019. Trata-se de um estudo epidemiológico descritivo e retrospectivo, com posterior análise dos dados, realizado através do Sistema de Informações Hospitalares (SIH/SUS) do período de 2008 a 2019, no Nordeste. A maioria das internações ocorreu entre pessoas pardas (82,75\%), da faixa etária dos 70-79 anos (26,93\%). Observou-se um crescimento dos custos de internação, durante o período, em 251,62\%, totalizando uma média anual de $37.750 .782,1$ reais. A taxa de letalidade no período foi de $17,38 \%$, diminuindo $14,28 \%$, relativamente, e o tempo de hospitalização aumentou $7,143 \%$, alcançando, em $2019,7,6$ dias. 0 número de internações aumentou $124,2 \%$ no período, e em média, o custo por internação foi de 1050,65 reais. Os estados com maior porcentagem de mortalidade e custo por internação foi Sergipe e Alagoas. Dessa forma, compreendeu-se que houve um aumento do número de internações e do custo total nesse período. Além disso, houve um importante aumento do custo por internação. Ademais, apesar do aumento do número de óbitos por ano, houve uma redução da taxa de letalidade.

\section{An analysis of costs and hospitalizations by cerebral vascular accident in the Northeast, 2008-2019}

\begin{abstract}
There is an important underfunding of the Unified Health System and a lack of information about health costs and demands, fundamental data for health managers to be able to make decision-making. To analyze stroke admissions to SUS in the Northeast region, from 2008 to 2019 . This is a descriptive and retrospective epidemiological study, with subsequent analysis of the data, carried out through the Hospital Information System (SIH/SUS) from 2008 to 2019, in the Northeast. Most hospitalizations occurred among brown people ( $82.75 \%)$, aged $70-79$ years (26.93\%). There was an increase in hospitalization costs, during the period, by $251.62 \%$, totaling an annual average of $37,750,782.1$ reais. The lethality rate in the period was $17.38 \%$, decreasing $14.28 \%$, relatively, and the hospitalization time increased $7.143 \%$, reaching 7.6 days in 2019 . The number of hospitalizations increased $124.2 \%$ in the period, and on average, the cost per hospitalization was 1050.65 reais. The states with the highest percentage of mortality and cost per hospitalization were Sergipe and Alagoas. Thus, it was understood that there was an increase in the number of hospitalizations and the total cost in this period. In addition, there was an important increase in the cost per hospitalization. Furthermore, despite the increase in the number of deaths per year, there was a reduction in the lethality rate.
\end{abstract}

Keywords: Stroke; Health management; Public administration.

Topic: Gestão Pública

Reviewed anonymously in the process of blind peer.
Received: 07/01/2021

Approved: 22/03/2021
Daniel Meira Nóbrega de Lima (iD

Universidade Federal da Paraíba, Brasil

http://lattes.cnpq.br/8706125054242800

http://orcid.org/0000-0001-8384-3895

danielmrnobrega@gmail.com

Gabriela Januário de Oliveira (ib

Universidade Federal da Paraíba, Brasil

http://lattes.cnpq.br/2797253186906526

http://orcid.org/0000-0003-2652-8455

januarigabriela@gmail.com

Herisson Rodrigues de Oliveira (iD)

Universidade Federal da Paraíba, Brasil

http://lattes.cnpq.br/2177128506824007

http://orcid.org/0000-0003-2273-5254

henri.md.92@gmail.com

\author{
Lucas Almeida de Souza (iD) \\ Centro Universitário de João Pessoa, Brasil \\ http://lattes.cnpq.br/6437212846352386 \\ http://orcid.org/0000-0003-2863-696X \\ lucasalmeidasouz@gmail.com \\ Maurus Marques de Almeida Holanda \\ Universidade Federal da Paraíba, Brasil \\ http://lattes.cnpq.br/2786546058482445 \\ http://orcid.org/0000-0003-2217-4434 \\ maurusholandaa@hotmail.com
}

Referencing this:

LIMA, D. M. N.; OLIVEIRA, G. J.; OLIVEIRA, H. R.; SOUZA, L. A.; HOLANDA, M. M. A.. Uma análise dos custos e internações por acidente vascular cerebral no Nordeste, 2008-2019. Revista Brasileira de Administração Científica, v.12, n.1, p.203-212, 2021. DOI: http://doi.org/10.6008/CBPC2179-684X.2021.001.0016 


\section{INTRODUÇÃO}

O acidente vascular cerebral (AVC) é uma das principais patologias tanto em países desenvolvidos como em desenvolvimento. Essa afecção de alta prevalência, ocasiona alta mortalidade, assim como diversas sequelas aos sobreviventes. Os principais fatores de risco para o AVC são a idade avançada, raça negra, sexo masculino, tabagismo atual ou pregresso, estado civil viúvo, hipertensão, diabetes mellitus e doença arterial coronariana (COPSTEIN et al., 2013; SANTOS et al., 2020).

Apesar dos graves impactos emocionais, sequelas motoras, funcionais e de comunicação, a assistência e as políticas de atenção às doenças cerebrovasculares ainda são insuficientes para a complexidade e demanda do tema. Ademais, observa-se uma importante deficiência no sistema de fiscalização e vigilância desses agravos, haja vista a falta de informações epidemiológicas acerca da real incidência e prevalência dessa entidade patológica (FONSECA et al., 2018).

Estima-se que $20 \%$ dos pacientes sobrevivem apenas ao primeiro mês e cerca de metade dos casos, sobrevivem por mais tempo com algum tipo de limitação ou grau de dependência. Compreende-se também que esses agravos são cada vez mais frequentes no Brasil, devido ao envelhecimento populacional e o aumento de expectativa de vida, sendo cada vez mais necessário o desenvolvimento de políticas públicas eficazes a essas patologias (OLIVEIRA et al., 2013).

Sendo assim, para reduzir a morbimortalidade do AVC no Brasil, deve-se melhorar a compreensão da população acerca do AVC, aumentar a resolutividade da atenção básica, qualificar o Serviço de Atendimento Móvel de Urgência para o atendimento desses profissionais, ampliar a oferta de leitos hospitalares e reorganizar a assistência secundária visando a promoção dos cuidados e necessidades dos pacientes após a alta hospitalar (BRASIL, 2011a)

O Plano de Ações Estratégicas para o Enfrentamento das Doenças Crônicas Não Transmissíveis (DCNT) no Brasil, 2011-2022, visa preparar o Brasil, para a transição epidemiológica e demográfica, que estamos vivendo. Esse plano é definido em três eixos: (I) Vigilância, informação, avaliação e monitoramento; (II) Promoção da Saúde; (III) Cuidado Integral. Entre eles, o primeiro eixo objetiva promover o desenvolvimento o desenvolvimento e implementação de políticas públicas, baseadas em informações de saúde (BRASIL, 2011b).

Uma vez que estamos passando por uma transição epidemiológica e demográfica, na qual há um aumento da expectativa de vida, envelhecimento populacional, substituição da carga de morbimortalidade de doenças transmissíveis para não-transmissíveis, modificação de uma situação de alta mortalidade para uma de alta morbidade, então os gastos em saúde estão cada vez maiores (OLIVEIRA et al., 2017).

Além disso, observa-se na realidade do Sistema Único de Saúde (SUS), um importante subfinanciamento, desigualdade do acesso a serviços, falta de tecnologias e apoio ao profissional de saúde. Assim, faz-se necessário uma melhoria da gestão e alocação dos recursos públicos, visando um sistema mais eficiente (SALDIVA et al., 2018).

Deste modo, a gestão de saúde atua em gerir os recursos, por meio de informações cruciais sobre os 
custos e recursos, visando a realização do processo decisório. Dessa forma, pesquisas epidemiológicas que discutam custos e demandas são essenciais para que gestores possam, eficientemente, estruturar e operacionalizar as melhores medidas possíveis (BRASIL, 2012).

O objetivo desse estudo foi analisar as internações por AVC no SUS na região Nordeste, durante o período de 2008 a 2019.

\section{METODOLOGIA}

\section{Delineamento do estudo}

O presente trabalho trata-se um de um estudo ecológico epidemiológico de caráter retrospectivo e descritivo, com análise de dados secundários de cunho documental, através das autorizações de internação hospitalar (AIH) devido a AVC do SUS.

\section{Campo de amostragem}

O foco do estudo foi a região Nordeste, que é subdividida em nove estados e 1.794 municípios. Essa região apresenta um território de 1.561 .177 km² e cerca de 57.071 .654 habitantes (IBGE, 2019).

\section{Fonte de dados}

Para o desenvolvimento da pesquisa, foram utilizados os registros compilados pelo Sistema de Informações Hospitalares (SIH). Os dados foram adquiridos através da plataforma do Departamento de Informática do Sistema Único de Saúde (Datasus), por meio do sistema de tabulação TABWIN (BRASIL, 2020).

\section{Amostragem}

A amostra do estudo trata-se dos casos de AVC, internados, durante o período de 2008 a 2019, no Nordeste, cadastrados no SIH. Assim, foi feito o levantamento do número de autorizações de internação hospitalar por AVC, sendo obtido cerca de 431.372 fichas.

O SIH registra e compila uma série de informações cruciais para gestores e profissionais de saúde, sendo a fonte oficial de dados do Ministério da Saúde (MS). Os dados foram obtidos por local de internação, empregando-se o capítulo do CID-10 (doença do aparelho circulatório) e lista de morbidade do CID-10 (acidente vascular cerebral não especificado hemorrágico ou isquêmico).

Foram excluídos os preenchimentos "sem informação" das fichas de AlH. Os critérios de inclusão do estudo foram todos os registros inclusos no SIH/SUS de internação por AVC, no Nordeste, durante o período de 2008 a 2019.

\section{Procedimentos metodológicos}

Os dados relativos as AlH por AVC no Nordeste foram coletadas, através da plataforma do Datasus, os dados estão atualizados até junho de 2020. 
As fichas de AlH compreendem um vasto número de informações, desde a raça e sexo do paciente até os custos da internação, tempo de permanência. Além disso, o Datasus disponibiliza as informações agrupadas por município, estado, região de residência ou internação, assim como ano e período. Dessa forma, foi utilizado o sistema de tabulação do Datasus (TABWIN) para a obtenção de cada variável utilizada.

Em seguida, as informações foram organizadas em quatro tabelas. A primeira tabela compilava as informações relativas a cor de pele/raça e faixa etária do paciente internado. A segunda tabela continha as informações acerca do número de internações e seus custos. A terceira tabela sintetizava os registros referentes aos dias de permanência e taxas de mortalidade. A quarta tabela abarcava os dados relativos aos estados.

Após a sistematização em tabelas, as informações foram realocadas no software Statistical Package for the Social Sciences (SPSS), versão 23.0, para ser realizado os cálculos de frequência para cor de pele/raça e faixa etária, assim como as contas de custo/internação. A variável custo/internação foi calculada dividindo o valor do custo total das internações por ano, nos estados e região, pelo número de internações hospitalares.

\section{Variáveis}

As variáveis empregadas foram subdividas em três grupos. O primeiro grupo avaliava o perfil sociodemográfico dos pacientes: cor de pele/raça (branca, preta, parda, amarela e indígena) e faixa etária (menor de 1 ano, 1-4 anos, 5-9 anos, 10-14 anos, 15-19 anos, 20-29 anos, 30-39 anos, 40-49 anos, 50-59 anos, 60-69 anos, 70-79 anos e 80 anos ou mais). O segundo grupo referia-se ao número de internações, os custos totais, o custo por internação, dias de permanência e a taxa de mortalidade. E o terceiro grupo abarcava os estados (Maranhão, Piauí, Ceará, Rio Grande do Norte, Paraíba, Pernambuco, Alagoas, Sergipe e Bahia) e a região Nordeste.

\section{Análise estatística}

Os dados organizados em tabelas foram realocados para o programa de software SPSS, versão 23.0. Por meio dele foram feitas as análises descritivas. O custo por internamento foi calculado empregando como numerado os custos totais e o denominador, o número de internações. Esta variável foi calculada por ano e período, em cada estado e toda região. Para as variáveis categóricas foi utilizado o cálculo de frequências absolutas e relativas.

\section{Considerações éticas}

Uma vez que a presente pesquisa fez uso de dados secundários de domínio público, fornecidos pelas instituições governamentais, de forma anônima. Além disso, não realizou pesquisas com animais ou humanos e está, de acordo, com a Resolução do Conselho Nacional de Saúde (CNS) n46, de 12 de dezembro de 2012. Assim, não foi necessário a submissão do projeto de pesquisa para a sua apreciação em Comitê de Ética e Pesquisa. 


\section{RESULTADOS}

Percebeu-se uma maioria do número de internações entre pessoas pardas $(82,75 \%)$, das faixas etárias $70-79$ anos (26,93\%), 80 anos ou mais (23,33\%) e 60-69 anos (23,01\%). 0 custo por internação é mais dispendioso entre amarelos $(1.060,29)$ e pardos $(1.025,61)$ e mais barato quanto mais avançado é a faixa etária.

Pessoas internadas por AVC da cor de pele preta $(7,3)$ e amarela $(7,3)$ passam mais dias internados, no geral. Verificou-se também, que as faixas etárias após os 50 anos, passavam menos tempo internado $(7,5)$. Registrou-se que uma maior porcentagem da mortalidade entre pardos $(16,07 \%)$ e pretos $(15,38 \%)$, assim como na faixa etária dos menores de 1 ano (22,99\%), 80 anos ou mais (22,76\%) e 70-79 anos (17,59\%) (Tabela $1)$.

Tabela 1: Internações hospitalares por AVC, no Nordeste, durante o período de 2008 a 2019, por raça/cor de pele e faixa etária.

\begin{tabular}{|c|c|c|c|c|}
\hline Variável & Internações & $\begin{array}{l}\text { Custo/Internação } \\
\text { (reais) }\end{array}$ & Dias de permanência & Taxa de mortalidade (\%/ano) \\
\hline \multicolumn{5}{|c|}{ Cor de pele/Raça } \\
\hline Branca & 24.787 & 840,83 & 6,7 & 14,91 \\
\hline Preta & 7.535 & 960,03 & 7,3 & 15,38 \\
\hline Parda & 198.612 & $1.025,61$ & 7,1 & 16,07 \\
\hline Amarela & 8.889 & $1.060,29$ & 7,3 & 14,76 \\
\hline Indígena & 198 & 593,34 & 6,3 & 13,01 \\
\hline \multicolumn{5}{|l|}{ Faixa etária } \\
\hline Até 1 ano & 87 & $1.177,94$ & 8,0 & 22,99 \\
\hline 1-4 anos & 112 & $1.965,94$ & 8,4 & 9,82 \\
\hline 5-9 anos & 180 & $2.290,92$ & 11,4 & 7,78 \\
\hline 10-14 anos & 531 & $1.557,81$ & 9,2 & 11,68 \\
\hline 15-19 anos & 1.492 & $1.346,21$ & 8,4 & 11,80 \\
\hline 20-29 anos & 5.402 & $1.300,81$ & 8,5 & 11,75 \\
\hline 30-39 anos & 12.929 & $1.226,15$ & 8,2 & 13,40 \\
\hline 40-49 anos & 32.115 & $1.155,04$ & 7,7 & 15,18 \\
\hline 50-59 anos & 63.616 & $1.100,45$ & 7,5 & 14,66 \\
\hline 60-69 anos & 99.251 & $1.079,79$ & 7,5 & 15,07 \\
\hline $70-79$ anos & 116.165 & $1.021,05$ & 7,5 & 17,59 \\
\hline $80+$ & 100.654 & 943,10 & 7,5 & 22,76 \\
\hline
\end{tabular}

Observou-se um crescimento dos custos de internação, durante o período, em 251,62\%. Em 2008, os gastos foram 15.462.788,15 reais e, em 2019, 54.370.363,27 reais. Além disso, não houve diminuição em nenhum ano, nesse período (Gráfico 1).

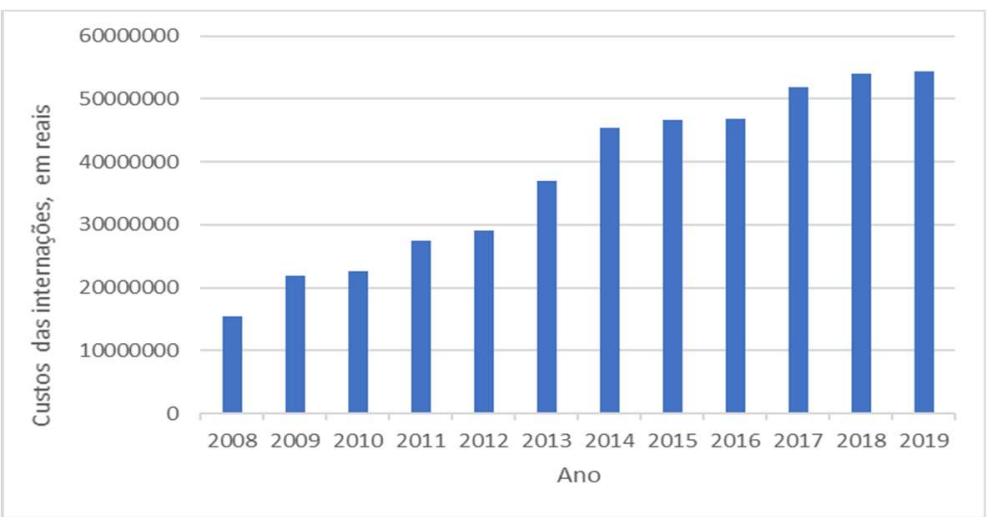

Gráfico 1: Evolução dos custos das internações por AVC, no Nordeste, durante o período de 2008 a 2019. 
Verificou-se que o sexo masculino se internou mais do que o sexo feminino, sendo responsável por 50,66\%. No entanto, em 2008, 2009 e 2013, notou-se uma predominância entre o sexo feminino (Gráfico 2).

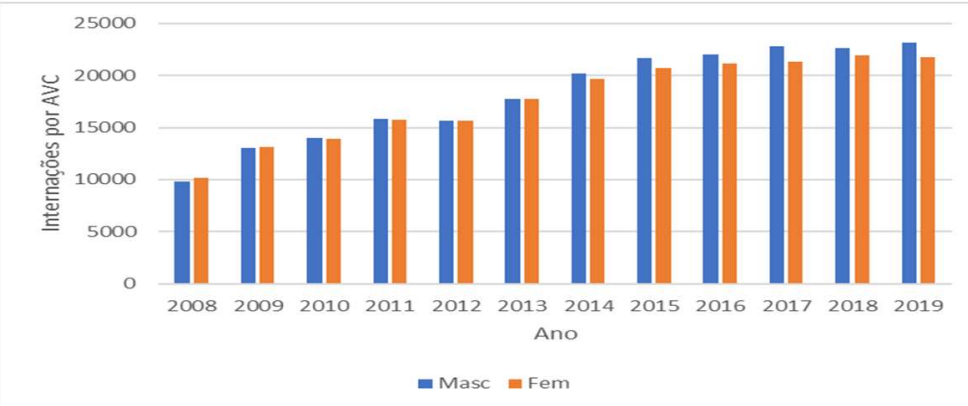

Gráfico 2: Internações por AVC, no Nordeste, entre 2008 a 2019, em ambos os sexos.

Os custos de internação durante o período de 12 anos, totalizou em 453.009.385,21 milhões de reais, e a média dos custos anual, foi de 37.750.782,1 reais. O número de internações, em 2008, foi de 20.017, e, em 2019, 44.877, assim houve um aumento de 124,2\% das internações anuais.

Averiguou-se um aumento do custo por internação, durante o período. Em 2008, a média dos custos era de 772,48 reais, passando em 2019, para 1211,54 reais. Deste modo, o custo por internação aumentou 56,84\% e a média anual, nesse período, foi de 1050,65 reais. Identificou-se também que os dias de permanência aumentaram em 7,143\%, no período e em média, os pacientes passaram 7,6 dias internados (Tabela 2).

Tabela 2: Custo das internações, internações/ano e custo/internação, no Nordeste, do período de 2008 a 2019.

\begin{tabular}{|c|c|c|c|c|}
\hline Ano & $\begin{array}{l}\text { Custo das internações por AVC } \\
\text { (reais) }\end{array}$ & Internações/Ano & $\begin{array}{l}\text { Custo/Internação } \\
\text { (reais) }\end{array}$ & Dias de permanência \\
\hline 2008 & $15.462 .787,15$ & 20.017 & 772,48 & 7,0 \\
\hline 2009 & $21.914 .930,87$ & 26.125 & 838,85 & 6,9 \\
\hline 2010 & $22.690 .542,62$ & 27.838 & 815,09 & 7,0 \\
\hline 2011 & $27.485 .444,45$ & 31.543 & 871,36 & 7,5 \\
\hline 2012 & $29.050 .758,90$ & 31.275 & 928,88 & 7,2 \\
\hline 2013 & $37.036 .007,54$ & 35.538 & $1.042,15$ & 7,4 \\
\hline 2014 & $45.466 .617,02$ & 39.880 & $1.140,09$ & 7,9 \\
\hline 2015 & $46.646 .114,13$ & 42.373 & $1.100,85$ & 7,8 \\
\hline 2016 & $46.908 .449,29$ & 43.121 & $1.087,83$ & 7,8 \\
\hline 2017 & $51.907 .032,031$ & 44.063 & $1.178,02$ & 7,9 \\
\hline 2018 & $54.070 .337,06$ & 44.522 & 1214,46 & 7,9 \\
\hline 2019 & $54.370 .363,27$ & 44.877 & 1211,54 & 7,5 \\
\hline Total & $453.009 .385,21$ & 431.172 & - & - \\
\hline Média & $37.750 .782,1$ & 35.931 & 1050,65 & 7,6 \\
\hline
\end{tabular}

O número de óbitos ao ano foi de 6.238 , ocorrendo um aumento de $92,25 \%$ desses eventos no período. A porcentagem da mortalidade diminuiu no período, reduzindo de 19,33\% para 16,57\%. Assim, houve um decréscimo de $2,76 \%$, da porcentagem absoluta, e $14,28 \%$, relativamente (Tabela 3 ).

Tabela 3: Óbitos/ano e taxa de mortalidade, no Nordeste, durante o período de 2008 a 2019.

\begin{tabular}{lll}
\hline Ano & Óbitos/Ano & Taxa de mortalidade (\%/ano) \\
\hline $\mathbf{2 0 0 8}$ & 3.869 & 19,33 \\
\hline $\mathbf{2 0 0 9}$ & 4.758 & 18,21 \\
\hline $\mathbf{2 0 1 0}$ & 4.824 & 17,33 \\
\hline $\mathbf{2 0 1 1}$ & 5.753 & 18,24 \\
\hline $\mathbf{2 0 1 2}$ & 5.776 & 18,47 \\
\hline $\mathbf{2 0 1 3}$ & 6.044 & 17,01 \\
\hline
\end{tabular}




\begin{tabular}{lll}
$\mathbf{2 0 1 4}$ & 6.628 & 16,62 \\
\hline $\mathbf{2 0 1 5}$ & 7.543 & 17,80 \\
\hline $\mathbf{2 0 1 6}$ & 7.516 & 17,43 \\
\hline $\mathbf{2 0 1 7}$ & 7.476 & 16,97 \\
\hline $\mathbf{2 0 1 8}$ & 7.236 & 16,25 \\
\hline $\mathbf{2 0 1 9}$ & 7.438 & 16,57 \\
\hline Média & 6.238 & 17,38 \\
\hline
\end{tabular}

A porcentagem da mortalidade foi menor em Pernambuco (14,32\%) e no Maranhão (14,38\%), e foi maior em Sergipe $(29,59 \%)$ e Alagoas $(25,15 \%)$. Os estados que apresentaram os menores custos por internação na região, foram o Piauí (753,59 reais) e o Maranhão (907,18 reais). E os estados com o maior custo, foram Sergipe (1.446,43 reais) e Alagoas (1.318,83 reais) (Tabela 4).

Tabela 4: Taxa de mortalidade e Custo/Internação, por estado do Nordeste, no período de 2008 a 2019.

\begin{tabular}{lll}
\hline Estado & Taxa de mortalidade (\%/ano) & Custo/Internação (reais) \\
\hline Maranhão & 14,38 & 907,18 \\
\hline Piauí & 15,47 & 753,59 \\
\hline Ceará & 17,89 & $1.015,50$ \\
\hline Rio Grande do Norte & 17,95 & $1.069,66$ \\
\hline Paraíba & 17,52 & $1.095,36$ \\
\hline Pernambuco & 14,32 & $1.219,76$ \\
\hline Alagoas & 25,15 & $1.318,83$ \\
\hline Sergipe & 29,59 & $1.446,43$ \\
\hline Bahia & 17,94 & 969,50 \\
\hline Nordeste & 17,38 & 1050,65 \\
\hline
\end{tabular}

\section{DISCUSSÃO}

A taxa de mortalidade varia conforme a etnia, classe econômica e gênero, deste modo, compreendese que ser negro é fator de risco para AVC (LOTUFO, 2005; SANTOS et al., 2020). No entanto, a presente pesquisa observou maior taxa de mortalidade entre a população parda, assim como estudo no Amapá (VAZ et al., 2020). Isso pode ser explicado pela maior identificação da população nordestina com essa cor de pele, uma vez que estudo em Maringá, verificou maior taxa de mortalidade em população branca, devido a sua composição étnica distinta (ARAÚJO et al., 2018). No Brasil, demonstrou-se uma maior prevalência entre pessoas brancas, seguida por pardas (RIBEIRO et al., 2016).

Percebeu-se que as faixas etárias acima dos 40 anos, apresentaram maior incidência dessas afecções, além disso, pessoas viúvas, de baixo nível socioeconômico e escolaridade possuem maiores riscos (COPSTEN et al., 2013). A idade média desses eventos, em estudo estadunidense foi de 68 anos para o sexo masculino e 72 anos para o sexo feminino (BEDAIWI et al., 2018).

Do mesmo modo, que a presente pesquisa registrou a maior média anual na faixa etária dos 70-79 anos (26,94\%), Dantas et al. (2019) também verificou esse resultado (26\%). Além disso, pacientes idosos tiveram maior taxa de letalidade. No entanto, houveram trabalhos que identificaram a maior parte das internações entre pessoas da faixa dos 60-69 anos (29,5\%), seguida pela dos 70-79 anos (27\%) (LENZ, 2018). Ademais, cerca de $50 \%$ dos casos ocorre entre pessoas acima de 70 anos (VAZ et al., 2020).

Estudo do Rio Grande do Sul (RS) constatou um importante aumento entre 2008 a 2017 de 155,75\% dos custos totais, entretanto no Nordeste, percebeu-se um aumento de $235,69 \%$ nesse período. Ademais, os custos por internação no RS são maiores, cerca de 1299,98 reais, em 2017, e apresentando um aumento de 
43,22\% no custo por internação, entre 2008 a 2017 (LENZ, 2018).

Segundo Abramczuk et al. (2009), o custo de um paciente vítima de AVC que se interna por três a cinco dias, isto é, uma internação curta, custa cerca de seiscentos e quarenta reais. Além disso, relatou que os custos para o SUS podem alcançar, dependendo da sequela, até trinta e dois mil reais, sendo em média seis mil reais. $\mathrm{O}$ tempo de internação pode ser até menos de 24 horas, sobretudo, quando evolui rápido ao óbito, ou até mesmo mais de 90 dias, quando desenvolve alguma complicação como escara ou pneumonia (NEVES et al., 2002).

Assim como o presente estudo, registrou uma discreta prevalência desses casos por pessoas do sexo masculino $(50,66 \%)$, outros estudos corroboraram este resultado verificando uma predominância neste sexo entre 50,61-62,3\% (PAULO et al., 2009; GARRITANO et al., 2012; BOTELHO et al., 2016; ARAÚJO et al., 2018; DANTAS et al., 2019; VAZ et al., 2020). Contudo, houve um estudo que demonstrou uma leve maioria entre o sexo feminino $(51,1 \%)$ (LENZ, 2019).

Nos Estados Unidos (EUA), também tem sido observado um aumento da incidência anual de AVC, no entanto, a porcentagem desses pacientes com necessidade de internação vem diminuindo (BEDAIWI et al., 2018). Quando, avaliado o Brasil, o número de internações aumentou 12,1\% entre 2009 a 2016, assim como o número de óbitos absolutos, entretanto, houve uma diminuição em $12,6 \%$ da taxa de mortalidade (DANTAS et al., 2019), sendo mais acentuada entre pessoas abaixo dos 70 anos (LOTUFO et al., 2017). E no RS, durante 2008 a 2017, houve um aumento do número de internações em 78,57\% e um decréscimo da porcentagem de mortalidade em 9,8\% (LENZ, 2019).

Enquanto o período de permanência hospitalizado dos pacientes pós-AVC no Nordeste foi de 7,6 dias, nos EUA, demonstrou-se que uma variação da média anual entre 4,9 a 5,6 dias, isto é cerca de 2 a 2,7 dias a menos. Ocorrendo uma leve diminuição ao longo do tempo, apesar de não ser significativo. Em estudo avaliando todo o Brasil, evidenciou-se uma quantidade levemente maior de permanência, cerca de 7,7 dias. Divergindo, sobretudo, nos extremos de idade, em que o Nordeste apresentava em maiores de 70 anos, cerca de -0,5 dias, em comparação à média nacional, entretanto, quando avaliado menores de 1 ano, o Nordeste demonstrou quase o triplo do tempo de permanência (BOTELHO et al., 2016). Em estado do sul do país, a média de permanência desses pacientes variou de 7,9 a 8,8 dias também acima da média nordestina (LENZ, 2019).

Averiguou-se que o período de hospitalização foi maior por AVC hemorrágico (AVCh) (17 dias +/- 16 dias) do que por AVC isquêmico (AVCi) (14 dias +/- 11 dias), assim como a taxa de letalidade, nos AVCh demonstrou 40,8\% e o isquêmico (14,9\%) (STAROSTKA-TATAR et al., 2018).

O diagnóstico de AVC em lactentes, muitas vezes, é demorado, bem como seu tratamento, acarretando em piores prognósticos. Entende-se que esse evento raro possui manifestação clínica pouco específica e que, frequentemente, há discrepância temporal entre o evento e o início dos sintomas. Dessa forma, é pesquisado outros diagnósticos diferenciais até que seja feita a suspeita de AVC (MEKITIAN e et al., 2009).

Além disso, ressalta-se que houve uma diminuição da porcentagem de mortalidade em cerca de 
$25,86 \%$, relativamente, em detrimento aos $14,28 \%$ no Nordeste. Ademais, enquanto a porcentagem de mortalidade no Nordeste era, em 2014, de $16,62 \%$, nos EUA era de 4,3\%, quase quatro vezes menor (BEDAIWI et al., 2018).

Uma vez que a maior parte das vítimas de AVC no Nordeste chegam ao pronto socorro e são atendidas, em mais de três horas, então o prognóstico desses pacientes é bastante desfavorável, consequentemente, observa-se uma maior porcentagem de mortalidade, assim como maior período de internação (MATOS, 2013, citado por BOTELHO, 2016).

Os custos por internação nos EUA, aumentaram 72,82\% entre 2006 e 2014, alcançando cerca de 48.299 dólares (BEDAIWI et al., 2018). Deste modo, o custo não apenas é muito mais elevado, em comparação, aos 1140,09 reais no Nordeste, como houve um aumento dos custos de forma mais acelerada, haja vista o acréscimo de 56,84\%, durante o período de 2008 a 2019 no Nordeste.

Identificou-se que, apesar da diminuição da taxa de mortalidade por AVC no Brasil, está tem ocorrido de forma desigual, uma vez que a região Nordeste ainda apresenta taxas mais elevadas, embora também tenha reduzido (RIBEIRO et al., 2016). O maior custo por internação nos estados de Sergipe e Alagoas podem ser explicados pela existência de quadros mais graves pela demora de assistência inicial.

As principais limitações desse trabalho referem-se aos preenchimentos incompletos das AlH, assim como a falta de detalhamento dos gastos. Outra limitação se dá pela incapacidade de identificar fatores de risco modificáveis e aspectos antropométricos, sendo necessário pesquisas coorte e transversais para a melhor compreensão.

\section{CONCLUSÕES}

A taxa de mortalidade varia, de acordo, com a etnia, gênero e faixa etária, sendo nesta região maior entre pessoas pardas e da faixa de idade menor de 1 ano, seguida por 80 anos ou mais. Verificou-se também que pessoas do sexo masculino se hospitalizaram mais. Pessoas negras e amarelas passam mais tempo internadas e, em média, o tempo de hospitalização no Nordeste foi de 7,6 dias, tendo aumentado 7,143\% no período.

Houve um aumento dos custos totais em $251,62 \%$ no período, e, em 2019 , alcançou $54.370 .363,27$ reais. Enquanto no mesmo período, houve um aumento de apenas $124,2 \%$. Deste modo, os custos por internação passaram de 772,48 reais, em 2008, para 1211,54 reais, em 2019, sendo em média, 1050,65 reais no período. Apesar do aumento do número de óbitos por ano, em 92,55\%, percebeu-se uma redução da taxa de mortalidade de $19,33 \%$ para $16,57 \%$. Ademais, a porcentagem de mortalidade foi menor em Pernambuco $(14,32 \%)$ e no Maranhão (14,38\%) e as mais altas em Sergipe (29,59\%) e Alagoas (25,15\%).

\section{REFERÊNCIAS}

ABRAMCZUK, B.; VILLELA, E.. A luta contra o AVC no Brasil. ComCiência, Campinas, n.109, 2009.

ARAÚJO, J. P.; DARCIS, J. V.; TOMAS, A. C.; MELLO, W. A. Mortality trend due to cerebrovascular accident in the City of Maringá, Paraná, between the years of 2005 to 2015.
International Journal of Cardiovascular Sciences, v.31, n.1, p.56-62, 2018. DOI: http://doi.org/10.5935/2359$\underline{4802.20170097}$

BEDAIWI, I. I.; ALFARAJ, S. Z.; PINES, J. M.. National trends in stroke and TIA care in U.S. emergency departaments and 
inpatient hospitalizations (2006-2014). Am. J. Emerg. Med., v.36, n.10, p.1870-1873, 2018. DOI:

http://doi.org/j.ajem.2018.08.013

BOTELHO, T. S.; MACHADO, C. D.; ARAÚJO, F. L.; ASSIS, S. C.. Epidemiologia do acidente vascular cerebral no Brasil. Temas em Saúde, v.16, n.2, p.361-377, 2016. DOI: http://doi.org/10.29327/213319

BRASIL. Ministério da Saúde. Departamento de Informática do Sistema Único de Saúde (Datasus) Informações de Saúde - TABNET. Assistência à saúde. Morbidade Hospitalar do SUS. Brasília: MS, 2020.

BRASIL. Ministério da Saúde. Secretaria de Atenção Especializada - Departamento de Atenção Especializada Linha de Cuidado do Acidente Vascular Cerebral (AVC) na Rede de Atenção às Urgências - 2011. Brasília: MS, 2011a.

BRASIL. Ministério da Saúde. Plano de Ações Estratégicas para o Enfrentamento das Doenças Crônicas Não Transmissíveis (DCNT) no Brasil, 2011-2022. Brasília: MS, 2011b.

BRASIL. Ministério da Saúde. Secretaria Executiva. Departamento de Economia da Saúde, Investimentos e Desenvolvimento. Glossário Temático: Economia da Saúde. 3 ed. Brasília: Ministério da Saúde, 2012.

COPSTEIN, L.; FERNANDES, J. G.; BASTOS, G. A.. Prevalence and risk factors for stroke in a population of Southern Brazil. Arquivos de Neuro-Psiquiatria, v.71, n.5, p,294-300, 2013. DOI: http://doi.org/10.1590/0004-282X20130024

DANTAS, L. F.; MARCHESI, J. F.; PERES, I. T.; HAMACHER, S.; BOZZA, F. A.; NEIRA, R. A.. Public hospitalizations for stroke in Brazil from 2009 to 2016. Plos One, v.14, n.3, e0213837, 2019. DOI:

http://doi.org/10.1371/journal.pone.0213837. eCollection $\underline{2019}$

FONSECA, A. R.; MURARI, R. S.; FONSECA, A. J.; BUENAFUENTES, S. M.. Impacto socioeconômico do acidente vascular cerebral no Estado de Roraima: Um estudo de coorte de base hospitalar. Revista Brasileira de Neurologia e Psiquiatria, v.22, n.2, p.124-141, 2018.

GARRITANO, C. R.; LUZ, P. M.; PIRES, M. L.; BARBOSA, M. T.; BATISTA, K. M.. Analysis of the mortality trend due to cerebrovascular accident in Brazil in the XXI century. Arquivos Brasileiros de Cardiologia, v.98, n.6, p.519-527, 2012. DOI: http://dx.doi.org/10.1590/S0066782X2012005000041

LENZ, G. S.. Acidente vascular cerebral: custos no SUS, no Rio Grande do Sul de 2007 a 2017. Monografia (Especialização) - Universidade Federal do Rio Grande do Sul, Esteio, 2018.

LOTUFO, P. A.. Stroke in Brazil: a neglected disease. São Paulo Medical Journal, v.123, n.1, p3-4, 2005. DOI: https://doi.org/10.1590/S1516-31802005000100001

LOTUFO, P. A.; GOULART, A. C.; PASSOS, V. M.; SATAKE, F.
M.; SOUZA, M. F.; FRANÇA, E. B.; RIBEIRO, A. L.; BENSEÑOR, I. J.. Cerebrovascular disease in Brazil from 1990 to 2015: Global Burden in Disease 2015. Revista Brasileira de Epidemiologia, v.20 n.1, p.129-141, 2017. DOI: http://doi.org/10.1590/1980-5497201700050011

MEKITIAN, C. E.; CARVALHO, W. B.. Acidentes vasculares encefálicos em pediatria. J. Pediatr., v.85, n.6, p.469-479, 2009. DOI: https://doi.org/10.1590/S002175572009000600002

NEVES, A. C.; FUKUJIMA, M. M.; JESUS, P. A.; FRANCO, C. M.; MOURA, R. C.; FONTES, S. V.; PRADO, G. F.. Custos do paciente com acidente vascular cerebral no setor de emergência do Hospital de São Paulo. Revista Neurociências, v.10, n.3, p.137-140, 2002. DOI: http://doi.org/10.4181/RNC.2002.10.137

OLIVEIRA, A. R.; ARAÚJO, T. L.; COSTA, A. G.; MORAIS, H. C.; SILVA, V. M.; LOPES, M. V.. Avaliação de pacientes com acidente vascular cerebral acompanhados por programas de assistência domiciliária. Revista da Escola de Enfermagem da USP, v.47, n.5, p.1143-1149, 2013. DOI: http://doi.org/10.1590/S0080-623420130000500019

OLIVEIRA, F. E.; SANTOS, A. S.; ROQUETE, F. F.. O uso das informações de custo em serviços públicos de urgência e emergência. Revista Eletrônica Gestão \& Saúde, v.8, n.2, p.230-253, 2017.

PAULO, R. B.; GUIMARÃES, T. M.; HELITO, P. V. P.; MARCHIORI, P. E.; YAMAMORO, F. I.; MANSUR, L. L.; SCAFF, M.; CONFORTO, A. B.. Acidente vascular cerebral isquêmico em uma enfermaria de neurologia: complicações e tempo de internação. Revista Associação Médica Brasileira, v.55, n.3, p.313-6, 2009.

RIBEIRO, A. L.; DUNCAN, B. B.; BRANT, L. C.; LOTUFO, P. A.; MILL, J. G.; BARRETO, S. M.. Cardiovascular health in Brazil. Circulation, v.133, n.4, p.422-433, 2016. DOI: https://doi.org/10.1161/CIRCULATIONAHA.114.008727

SALDIVA, P. H.; VERAS, M.. Gastos públicos com saúde: breve histórico, situação atual e perspectivas futuras. Estudos Avançados, v.32, n.92, 47-60, 2018. DOI: http://doi.org/10.5935/0103-4014.20180005

SANTOS, L. B.; WATERS, C.. Perfil epidemiológico dos pacientes acometidos por acidente vascular cerebral: Revisão Integrativa. Brazilian Journal Development, v.6, n.1, 2020. DOI: https://doi.org/10.34117/bjdv6n1-198

STAROSTKA-TATAR, A.; LABUZ-ROSZAK, B.; SKRZYPEK, M.; LASEK-BAL, A.; GASIOR, M.; GIERLOTKA, M.. Characteristics of hospitalizations due to acute stroke in the Silesian Province, Poland, between 2009 and 2015. Neurol Neurochir Pol., v.52, n.2, p.252-262, 2018. DOI: http://doi.org/10.1016/j.pjnns.2017.11.010

VAZ, D. W.; EVANGELISTA, H. I.; PONTES, L. C.; SILVA, J. B.; REZENDE, R. W.; ACATAUASSÚ, L. P.. Epidemiological profile of stroke in the State of Amapá, Brazil. Research, Society and Development, v.9, n.8, e938986642, 2020. DOI: https://doi.org/10.33448/rsd-v9i8.6642

A CBPC - Companhia Brasileira de Produção Científica (CNPJ: 11.221.422/0001-03) detém os direitos materiais desta publicação. Os direitos referem-se à publicação do trabalho em qualquer parte do mundo, incluindo os direitos às renovações, expansões e disseminações da contribuição, bem como outros direitos subsidiários. Todos os trabalhos publicados eletronicamente poderão posteriormente ser publicados em coletâneas impressas sob coordenação da Sustenere Publishing, da Companhia Brasileira de Produção Científica e seus parceiros autorizados. Os (as) autores (as) preservam os direitos autorais, mas não têm permissão para a publicação da contribuição em outro meio, impresso ou digital, em português ou em tradução. 